\title{
Stirring by squirmers
}

\author{
Z H I L I N ${ }^{1}$, J E A N - L U C THIF F E A U L T $\mathbf{T}^{1,2}$, \\ ANDSTEPHEN CHILDRES S \\ ${ }^{1}$ Institute for Mathematics and Applications, University of Minnesota - Twin Cities, 207 \\ Church Street S.E., Minneapolis, MN 55455, USA \\ 2 Department of Mathematics, University of Wisconsin - Madison, 480 Lincoln Drive, \\ Madison, WI 53706, USA \\ ${ }^{3}$ Courant Institute of Mathematical Sciences, New York University, 251 Mercer Street, \\ New York, NY 10012, USA
}

\begin{abstract}
We analyse a simple 'Stokesian squirmer' model for the enhanced mixing due to swimming micro-organisms. The model is based on a calculation of Thiffeault \& Childress [Physics Letters A 374, 3487 (2010)], where fluid particle displacements due to inviscid swimmers are added to produce an effective diffusivity. Here we show that for the viscous case the swimmers cannot be assumed to swim an infinite distance, even though their total mass displacement is finite. Instead, the largest contributions to particle displacement, and hence to mixing, arise from random changes of direction of swimming and are dominated by the far-field stresslet term in our simple model. We validate the results by numerical simulation. We also calculate nonzero Reynolds number corrections to the effective diffusivity. Finally, we show that displacements due to randomly-swimming squirmers exhibit PDFs with exponential tails and a short-time superdiffusive regime, as found previously by several authors. In our case, the exponential tails are due to 'sticking' near the stagnation points on the squirmer's surface.
\end{abstract}

\section{Introduction}

Swimming creatures affect their environment in many ways, and one which has received attention recently is how they mix the surrounding fluid. This phenomenon is called biogenic mixing or biomixing. The most striking and controversial setting is the ocean: Dewar et al. (2006) suggested that marine life might have an impact on vertical mixing in the ocean.

Katija \& Dabiri (2009) proposed that the dominant effect involved in biomixing is the mass displacement due to a swimming body. This phenomenon is called Darwinian drift, after Darwin (1953), though the displacement due to a moving cylinder was obtained by Maxwell (1869). Thiffeault \& Childress (2010) derived the effective diffusivity of an 'ideal gas' of randomly-distributed non-interacting swimmers, and showed that it depends on the induced squared-displacement of fluid particles by the swimmers, as opposed to the net mass displaced.

In the present paper we apply the techniques of Thiffeault \& Childress (2010) to swimmers in the low-Reynolds number regime. Since the experiments of Wu \& Libchaber (2000), there has been considerable work in that direction. Two general types of swimmers arise: pushers or pullers, depending on whether the propulsion takes place ahead or behind the swimmer's centre of mass. Several authors (Dombrowski et al. 2004; Hernandez-Ortiz et al. 2006; Saintillian \& Shelley 2007; Underhill et al. 2008) have pointed out that at high volume fractions the pushers align spontaneously with their neighbours, producing 


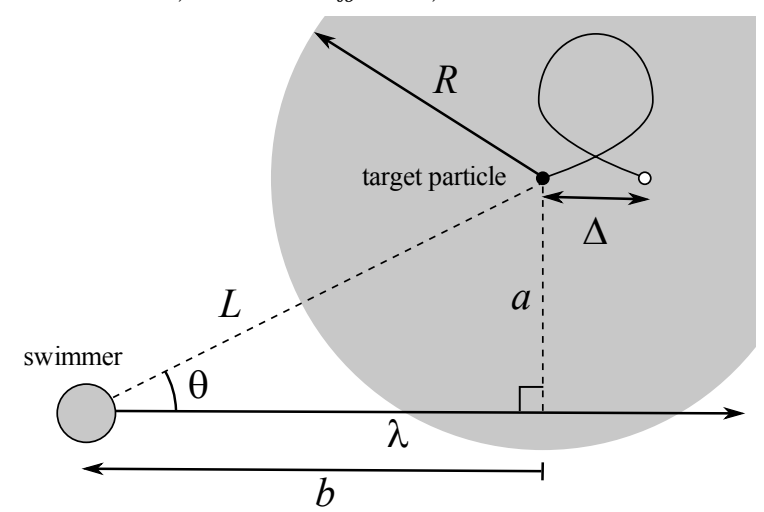

FiguRE 1. Definition of impact parameters $a$ and $b$, displacement $\Delta=\Delta_{\lambda}(a, b)$, and swimming path length $\lambda$. In this picture the parameter $b$ is positive; negative $b$ corresponds to the swimmer starting its trajectory past the point of initial closest approach. The filled dot is the initial position of the target particle and the hollow dot is its final position after the swimmer has moved by a distance $\lambda$. The 'interaction disk' of radius $R$ is also shown.

large-scale motions. At low volume fractions, pushers and pullers behave similarly, moving in a relatively uncorrelated manner. It is this limit that we treat in this paper. We use Lighthill (1952) and Blake (1971)'s 'squirmer' model, where a swimmer is modelled as a sphere in Stokes flow with a prescribed tangential velocity distribution. These have recently been studied by Ishikawa \& Pedley (2007) and Drescher et al. (2009) as models of the green alga Volvox. Squirmers could also be appropriate for Chlamydomonas (Leptos et al. 2009; Guasto et al. 2010; Drescher et al. 2010), as the observed velocity fields resemble those of the models described here. We find a formula for the effective diffusion coefficient due to the squirmers, and show that it depends predominantly on the far-field displacements imposed by the swimmer. Moreover, the dominant contributions arise from the turning motion of the swimmers, in sharp contrast to the potential flow case (Thiffeault \& Childress 2010) where the path length of straight swimming could be considered infinite for all practical purposes. We also show that the probability distribution function of displacements due to squirmers has exponential tails, as in Leptos et al. (2009), and that it exhibits a short-time superdiffusive regime as in Wu \& Libchaber (2000).

The formula we derive for the effective diffusivity has a wide applicability beyond squirmers, for instance to swimmers at larger Reynolds numbers with wakes. In that spirit, we derive finite (but small) Reynolds number corrections to the effective diffusivity using the Oseen form for the far field, and find that the effective diffusivity falls off as $R e^{-0.61}$ from its Stokes (zero $R e$ ) value.

\section{Computing the effective diffusivity}

We first consider a single swimmer in two or three dimensions that moves in a straight line at constant speed $U$ for a distance $\lambda$. For simplicity, the swimmer is assumed axially symmetric with characteristic size $\ell$, and moves along its axis of symmetry. We are concerned with the effect of the swimmer on a target Lagrangian particle. There are two 'impact parameters' necessary to describe such a situation: the initial perpendicular distance to the particle, $a \geqslant 0$, and the relative distance $b$ of the start of the trajectory to the point of initial closest approach (see figure 1). Note that $b$ may be positive or negative, and is positive if closest approach would occur at a positive time. After the swimmer traverses its full path length $\lambda$, the target particle is displaced by a distance $\Delta_{\lambda}(a, b)$. 
Inspired by Einstein's theory of Brownian motion (Einstein 1956) and following Thiffeault \& Childress (2010), we now model the displacement of the target particle after $M$ encounters with swimmers as a series of 'kicks', each of which moves the particle by $\Delta_{\lambda}\left(a_{k}, b_{k}\right)$ in some random direction $\hat{\boldsymbol{r}}_{k}$. The position $\boldsymbol{x}_{M}$ of the particle is

$$
\boldsymbol{x}_{M}=\boldsymbol{x}_{0}+\sum_{k=1}^{M} \Delta_{\lambda}\left(a_{k}, b_{k}\right) \hat{\boldsymbol{r}}_{k}
$$

and we will assume $\boldsymbol{x}_{0}=0$. The swimmers are assumed identical and non-interacting, and the encounters are taken to be statistically independent. Their directions of swimming are assumed isotropically distributed. In this case, the mean square displacement after $M$ such encounters is given by

$$
\left\langle\left|\boldsymbol{x}_{M}\right|^{2}\right\rangle=\sum_{k=1}^{M}\left\langle\Delta_{\lambda}^{2}\left(a_{k}, b_{k}\right)\right\rangle=M\left\langle\Delta_{\lambda}^{2}(a, b)\right\rangle,
$$

because the $a_{k}$ and $b_{k}$ are identically distributed for each encounter, with the brackets denoting an average over $a$ and $b$. (We could also average over distributions of $\lambda$ and $U$, but we assume constant values for simplicity.) Our theory will apply in the dilute limit, which we will define more carefully below.

The displacement $\Delta_{\lambda}$ is assumed small compared to $\lambda$, so that we can regard the target particle as approximately fixed during an encounter. We assume that each swimmer advances by a fixed length $\lambda$ and then changes direction randomly, so after a time $t$, $U t / \lambda$ such direction changes have occurred. For the $2 \mathrm{D}$ case, the number of swimmers in a ring of radius $L$ and thickness $\mathrm{d} L$ around the target particle is $2 \pi n L \mathrm{~d} L$, where $n$ is the number density of swimmers. To define an encounter, we introduce an 'interaction disk' (sphere in 3D) of radius $R$ around the target particle (see figure 1), and consider an encounter as occurring when a swimmer enters that disk. If a swimmer does not enter the interaction disk, then the motion of the target particle is negligible. The interaction disk allows us to be more precise about what we mean by a dilute suspension of swimmers: we require $n R^{2} \ll 1\left(n R^{3} \ll 1\right.$ in $\left.3 \mathrm{D}\right)$ so that encounters are isolated from each other.

The expected number $M$ of such encounters after a time $t$ is best written in terms of integrals over $a=|L \sin \theta|$ and $b=L \cos \theta$ :

$$
M=\frac{2 n U t}{\lambda} \int_{0}^{R} \int_{-\sqrt{R^{2}-a^{2}}}^{\lambda+\sqrt{R^{2}-a^{2}}} \mathrm{~d} b \mathrm{~d} a=2 n R U t+\pi n U t \frac{R^{2}}{\lambda} .
$$

For large path length $\lambda \gg R$ the final term can be neglected, and we obtain the same expression as in Thiffeault \& Childress (2010), where an infinite path length was assumed: for large path length, the frequency of encounters is independent of $\lambda$.

Next we compute the average squared displacement (2.2) by integrating $\Delta_{\lambda}^{2}$ :

$$
M\left\langle\Delta_{\lambda}^{2}\right\rangle=\frac{2 n U t}{\lambda} \int_{0}^{R} \int_{-\sqrt{R^{2}-a^{2}}}^{\lambda+\sqrt{R^{2}-a^{2}}} \Delta_{\lambda}^{2}(a, b) \mathrm{d} b \mathrm{~d} a .
$$

We assume that $\Delta_{\lambda}(a, b)$ decays rapidly for large $a \gg \ell$, where $\ell$ is the characteristic size of a swimmer. In that case we can replace $R \gg \ell$ by infinity; after also replacing $\boldsymbol{x}_{M}$ by $\boldsymbol{x}(t)$ we obtain

$$
\left\langle|\boldsymbol{x}(t)|^{2}\right\rangle=\frac{2 n U t}{\lambda} \int_{0}^{\infty} \int_{-\infty}^{\infty} \Delta_{\lambda}^{2}(a, b) \mathrm{d} b \mathrm{~d} a=: 4 \kappa t \quad(2 \mathrm{D}),
$$

where we introduced the effective diffusivity $\kappa$. In 3D the analogous expression can easily 
shown to be

$$
\left\langle|\boldsymbol{x}(t)|^{2}\right\rangle=\frac{2 \pi n U t}{\lambda} \int_{0}^{\infty} \int_{-\infty}^{\infty} a \Delta_{\lambda}^{2}(a, b) \mathrm{d} b \mathrm{~d} a=: 6 \kappa t \quad(3 \mathrm{D}) .
$$

Note that the 'interaction disk' of radius $R$ has disappeared from (2.5) and (2.6): because of the rapid decay of $\Delta_{\lambda}(a, b)$ with $a$, we are free to overcount encounters when computing $\left\langle|\boldsymbol{x}(t)|^{2}\right\rangle$ because faraway encounters hardly displace the target particle.

Thiffeault \& Childress (2010) discussed models based upon potential flow past a cylinder or a sphere and made use of an approximate form of (2.5)-(2.6). The approximate form effectively assumes that the particle doesn't move unless the swimmer actually crosses the point of initial closest approach (a good approximation for potential flow, see figure $4(\mathrm{a}))$. Thus $\Delta_{\lambda}(a, b)$ takes the 'top-hat' form

$$
\Delta_{\lambda}(a, b)= \begin{cases}\Delta(a), & \text { if } 0<b<\lambda \\ 0, & \text { otherwise }\end{cases}
$$

This approximation works very well for potential flow. With the approximate form (2.7), the effective diffusivities defined by (2.5)-(2.6) become

$$
\kappa= \begin{cases}\frac{1}{2} U n \int_{0}^{\infty} \Delta^{2}(a) \mathrm{d} a, & (2 \mathrm{D}), \\ \frac{\pi}{3} U n \int_{0}^{\infty} a \Delta^{2}(a) \mathrm{d} a, & (3 \mathrm{D}),\end{cases}
$$

as in Thiffeault \& Childress (2010). Because the potential flow approximation is inappropriate for micro-organisms, we will not use (2.8) but rather take the full form (2.5)-(2.6).

Note that the number density scaling of (2.5)-(2.6) was derived by Underhill et al. (2008) using the Green-Kubo formula, but here we get explicit values for the prefactor. As we will see below, this allows us to determine where the dominant contribution to $\kappa$ arises from. In addition, the dimensionless prefactor can vary significantly depending on the swimmer under consideration.

\section{Stirring by squirmers}

Squirmers are a simple model of swimming micro-organisms in the Stokesian regime. Lighthill (1952) first introduced the model and Blake (1971) later extended the analysis. Recently, the squirmer model has been applied to describe the behaviour of slow-moving ciliates (see for example Ishikawa et al. 2006; Ishikawa \& Pedley 2007; Drescher et al. 2009), whose motion is propelled by arrays of pulsating cilia on their surface. A squirmer is generally a three-dimensional sphere moving in a Stokesian fluid with velocity field prescribed on its boundary. The velocity field generated by such a squirmer is derived by an expansion of solutions obtained by separation of variables. The steady swimming imposes a force-free condition between the fluid and the body which in turn translates to algebraic equations for the expansion coefficients of the solution.

Following Ishikawa et al. (2006), we choose a specific instance of an axially-symmetric squirmer of radius $\ell$, swimming along the positive $z$-axis at a constant speed $U$. Its free-space, steady, axisymmetric streamfunction in a comoving reference frame is

$$
\psi(\rho, z)=\frac{1}{2} \rho^{2} U\left(-1+\frac{\ell^{3}}{r^{3}}+\frac{3 \beta \ell^{2} z}{2 r^{3}}\left(\frac{\ell^{2}}{r^{2}}-1\right)\right)
$$

in cylindrical coordinates where $r=\sqrt{\rho^{2}+z^{2}}=\sqrt{x^{2}+y^{2}+z^{2}}$. The stresslet coefficient $\beta$ is a free, dimensionless parameter and the special case $\beta=0$ corresponds to a sphere in potential flow; we shall take $\beta=5$ in numerical examples as in Ishikawa et al. (2006). 


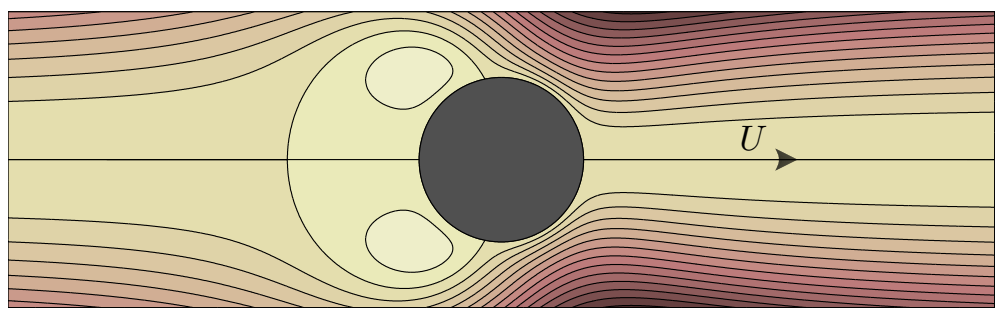

FIGURE 2. Streamfunction contours for a steady squirmer with $\beta=5$, in a comoving reference frame. Note the wake or closed 'bubble' behind the squirmer.

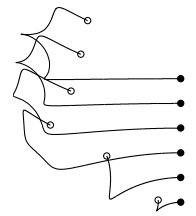

$b / \lambda=1$

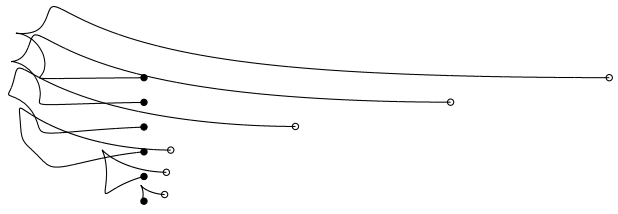

$b / \lambda=0.05$

FiguRE 3. Typical trajectories of a target particle in the fixed lab frame for different values of $a$ and $b$ due to a single squirmer moving from left to right, with $\ell=U=1, \lambda=100$ and $\beta=5$. The trajectories are offset vertically for clarity. The initial positions of the particle are marked by solid dots and the final positions by hollow dots. In each panel, solid dots from top to bottom correspond to $\log (a / \ell)=2,1,0,-1,-2,-3$.

Figure 2 shows the contours of the streamfunction (3.1) for a squirmer swimming from left to right, and figure 3 shows typical trajectories of a passive target particle displaced by the squirmer with different values of the impact parameters $a, b$. These types of trajectories were discussed by Dunkel et al. (2010) in the context of swimming organisms.

Compared with the fore-aft symmetry of the potential flow case $\beta=0$, a squirmer with $\beta>0$ has a downstream wake or 'bubble', which we will discuss in section 4 . In addition, a passive particle displaced by the squirming motion experiences a much larger excursion when the swimmer is still more than several body lengths away from the point of initial closest approach, while in potential flow this contribution is negligible to the total displacement. This suggests that the finite-path displacement function $\Delta_{\lambda}(a, b)$ due to a squirmer has a more complicated dependence on the details of the swimming path through the values of $a, b$, and $\lambda$. A direct consequence of this feature is that the diffusivity integral can no longer be reduced to one dimension by restricting the integration domain of $b$ as in equation (2.7). Instead, the full two-dimensional integral (2.6) has to be computed.

Thiffeault \& Childress (2010) showed that the integrals (2.5) and (2.6) for potential swimmers are dominated by "head-on" collisions, namely, significant particle displacement only occurs when the impact parameter $a \lesssim \ell$ and when the swimmer passes by the particle. We next investigate the dominant contribution of the integral for the squirmers (2.6). Transforming the integral as

$$
\lambda^{-1} \ell^{-4} \int_{-\infty}^{\infty} \int_{0}^{\infty} a \Delta_{\lambda}^{2}(a, b) \mathrm{d} a \mathrm{~d} b=\int_{\mathbb{R}^{2}} \ell^{-4} a^{2} \Delta_{\lambda}(a, b) \mathrm{d} \log (a / \ell) \mathrm{d}(b / \lambda),
$$

we compare in figure 4 the spatial distributions of the dimensionless integrand $\ell^{-4} a^{2} \Delta_{\lambda}(a, b)$ due to a sphere in potential flow and a squirmer in Stokes flow, for $\ell=U=1$ and $\lambda=100$, in the $\log (a / \ell)-(b / \lambda)$ plane. The far-field stresslet-only approximation to the displacement for a squirmer is also plotted as an inset in figure 4(b). The inset plot is almost identical to the full plot for any $\lambda \gg \ell$. Note that there is a vertical strip near $b=0$ in 


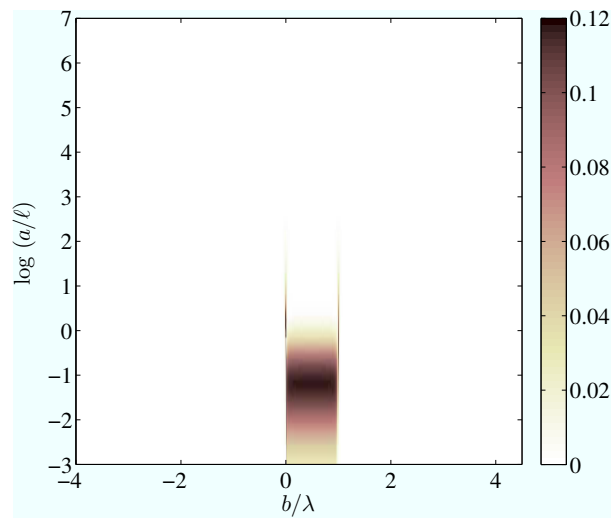

(a)

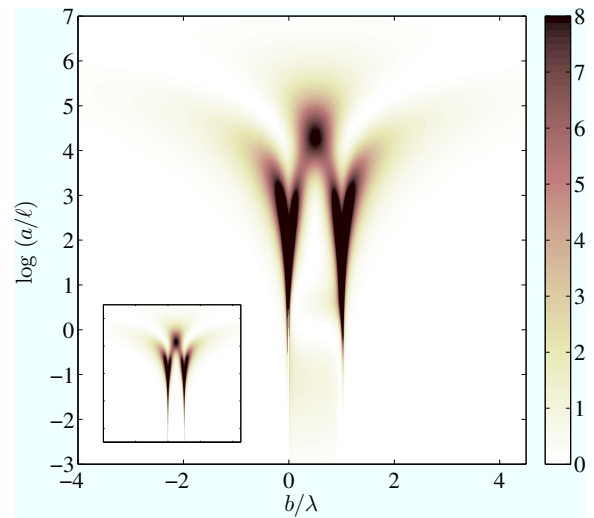

(b)

FiguRE 4. Integrand $\ell^{-4} a^{2} \Delta_{\lambda}^{2}(a, b)$ from (3.2) due to (a) potential flow past a sphere; (b) a squirmer with $\beta=5$; the near-field potential-flow pattern can be dimly seen for $\log a<0$, but is much weaker than the far field contribution. Inset: integrand corresponding to the far-field approximation of the squirmer flow. In all figures, $\ell=U=1$ and $\lambda=100$.

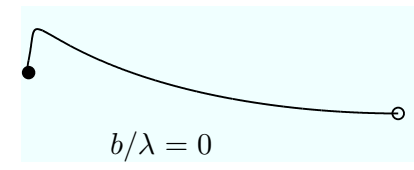

FiguRE 5. Trajectories of target particle for $a / \ell=1.1$ and different values of $b$. The filled dot is the initial position of the target particle and the hollow dot is its final position after the swimmer has moved by a distance $\lambda=100$ to the right. The net displacement is small at $b=\lambda / 2$ because of cancellation as the particle moves towards and then follows the swimmer.

each figure which corresponds to the situation where the initial particle position is inside the swimmer or its wake and is therefore excluded in the calculations. We shall return to the effect of particles trapped in the wake in section 4.

For a sphere in potential flow, the dominant contributions to (3.2) come from a rectangle (figure 4(a)) where the values of the integrand are nearly uniform in $b$ for $b \in[0, \lambda]$. This justifies the approximation (2.7). However, for a squirmer the dominant contributions to the integral (3.2) come from a much larger and irregular region with $a / \ell \gtrsim 1$. The contribution from this region is negligible in the potential case.

What explains the strong peaks in figure 4 (b) along the lines $b / \lambda=0, b / \lambda=1$ ? Figure 5 provides the answer: a target particle with $b / \lambda=1 / 2$ undergoes a sizable excursion, but has a small net displacement since it moves towards and then follows the squirmer, giving back a large part of the displacement it first underwent. In contrast, for $b / \lambda=0$ and $b / \lambda=1$ the squirmer starts or stops (i.e., changes direction) before the particle reverses direction and gives back its displacement.

Now that we know where the contributions to (3.2) arise from, we apply the basic theory to the squirmer model and find

$$
\kappa=57.0 U n \ell^{4}, \quad \lambda \gg \ell
$$

which is more than 200 times bigger than the effective diffusivity due to potential swimmers under the same set of parameters. One source of this large enhancement comes from the free parameter $\beta$, equal to 5 here. Since at far field the dominant perturbation to the uniform flow in the stream function (3.1) is multiplied by $\beta$, we expect that for large $\beta, \kappa \approx 2.1 \beta^{2} U n \ell^{4}$ (the far-field value, with coefficient fitted in figure $6(\mathrm{a})$ ), whereas 


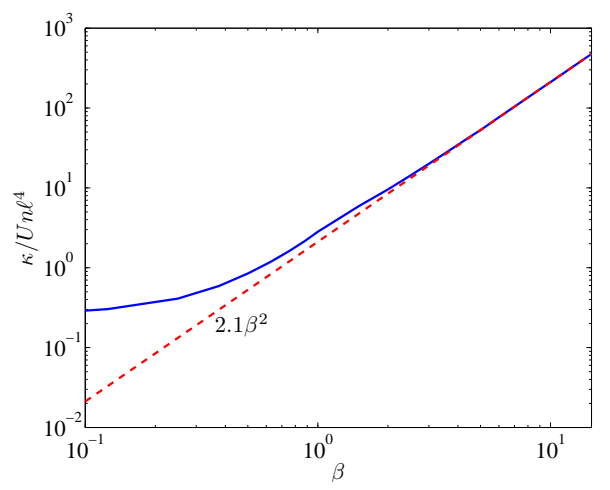

(a)

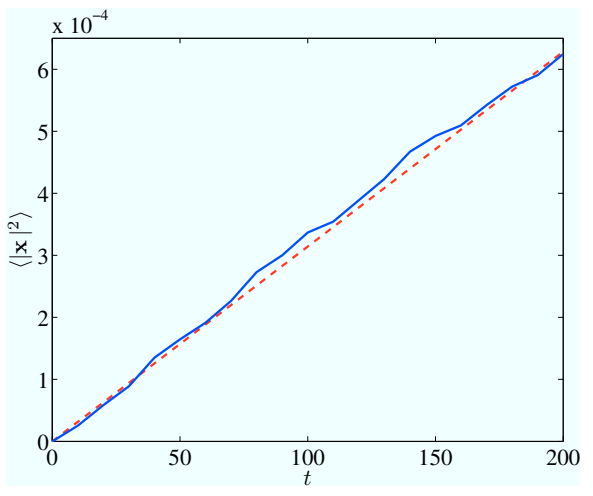

(b)

FiguRE 6. (a) Dependence of effective diffusivity on $\beta$ for $\lambda \gg \ell$ : the solid line is the numerical integration of (2.6) with $\Delta_{\lambda}$ computed with the streamfunction (3.1). For large $\lambda, \kappa$ is independent of $\lambda$. The dashed line is the effective diffusivity due only to the stresslet term. (b) The mean-squared displacement (solid line) of a target particle for $2 \times 10^{6}$ realisations of 10 squirmers, with $\ell=U=1$. The dashed line is the squared-displacement predicted by (3.3), using a number density $n=10^{-8} \ell^{-3}$.

the diffusivity converges to the potential value $0.266 U n \ell^{4}$ as $\beta \rightarrow 0$. Figure 6 (a) shows how different values of $\beta$ change the effective diffusivity $\kappa$. Using the far-field form of our model, we can recover the diffusivity $1.53 \mu \mathrm{m}^{2} / \mathrm{sec}$ measured by Leptos et al. (2009), for volume fraction $1.6 \%$, velocity $U=100 \mu \mathrm{m} / \mathrm{sec}$, and size $\ell=5 \mu \mathrm{m}$, using a stresslet amplitude $\beta=0.6$. The linearity of the diffusivity in the velocity and volume fraction (for dilute suspensions) has been pointed out by Underhill et al. (2008) and Leptos et al. (2009), but is verified here from first principles with computed prefactors.

Next we validate the results with direct simulations conducted as in Thiffeault \& Childress (2010). For each trial, we use 10 randomly-distributed, non-interacting squirmers with $\ell=U=1$ moving in random initial directions in a large periodic box of size 1000 . Each squirmer moves in a straight line for a length $\lambda=100$ and then turns in a new random direction. The turning times are randomized so that the squirmers do not all turn simultaneously. The displacement statistics are identical when this asynchrony is relaxed. The target particle initially at the origin is displaced by the superposition of the flows created by each squirmer (this is a good approximation at such low densities). Figure 6(b) shows the mean-squared displacement $\left\langle|\boldsymbol{x}|^{2}\right\rangle$ of a target particle over $2 \times 10^{6}$ trials (realisations). The agreement between the solid curve (numerically-simulated $\left\langle|\boldsymbol{x}|^{2}\right\rangle$ ) and the dashed line ( $6 \kappa t$ with $\kappa$ computed with the integral (2.6)) verifies the the theoretical prediction. We also simulated a large number of particles advected by a single realisation of 30 squirmers: the squared-displacement averaged over all the particles obeys the theory for large times, as expected.

\section{Wake transport}

In the previous section we have ignored the wake or closed 'bubble' behind the squirmer, visible in figure 2, since it is helpful to treat it separately. Assume that a particle is initially trapped in the swimmer's wake: it will then be displaced by the full swimmer's path length $\lambda$, until the swimmer changes direction and new particles are introduced in the wake. We can thus approximate the displacement $\Delta_{\lambda}$ to be equal to $\lambda$ if the particle is inside the wake. The wake contribution to the effective diffusivity is then obtained 


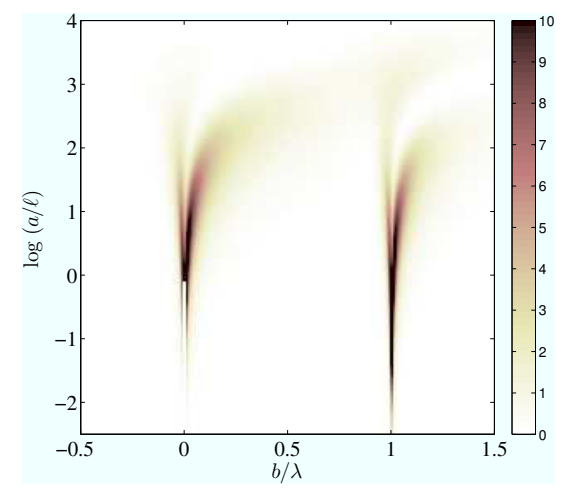

(a)

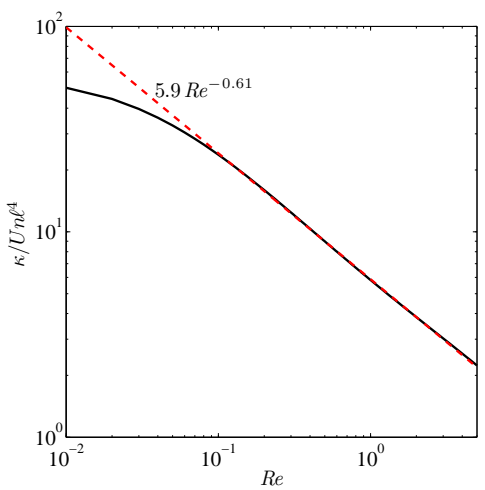

(b)

Figure 7 . The role of the Reynolds number Re. (a) Integrand $\ell^{-4} a^{2} \Delta_{\lambda}^{2}(a, b)$ from (3.2) for displacements due to the far-field Oseen flow (5.1), for $R e=0.5$ and $\lambda=100$. (b) The effective diffusivity $\kappa$ from (2.6) as a function of $R e$. For small $R e$ we recover (3.3).

from $(2.6)$ as

$$
\kappa_{\text {wake }}=\frac{U n}{6 \lambda} \lambda^{2} \int_{\text {wake }} 2 \pi a \mathrm{~d} a \mathrm{~d} b=\frac{1}{6} U n V_{\text {wake }} \lambda
$$

where $V_{\text {wake }}$ is the volume of the wake 'bubble'. Note that $n V_{\text {wake }}$ is the total volume fraction of wake bubbles in the fluid. The wake effective diffusivity (4.1) is potentially much larger than that due to displacements outside the wake bubble, since (4.1) is directly proportional to $\lambda$. In practice, many swimmers do not exhibit a wake bubble, and even for those that do the time-dependence of the velocity field near the swimmer and the effect of other swimmers can cause particles to jump in and out of the wake, which may lower (4.1) considerably. Molecular diffusivity will also limit the path length over which material remains trapped in the wake: for $\lambda \gtrsim U V_{\text {wake }}^{2 / 3} / \kappa_{\text {molecular }}$, significant amounts of material will have leaked out.

\section{Nonzero Reynolds number}

In the previous section, the basic theory was applied to model squirmer-induced mixing in Stokes flow, for which the Reynolds number $R e=0$ and inertial effects are neglected. We identified the dominant contributions as arising from the far field (stresslet). To generalise the squirmer far field and to account for finite (but small) Reynolds number effects on diffusion, we consider the analogous problem for the Oseen equations, which linearise the inertial forces (Oseen 1910). The singularity analogous to the squirmer farfield has streamfunction

$$
\psi=-\frac{1}{2} U \rho^{2}+\frac{U \ell^{2} \rho^{2}}{r^{2}}\left(e^{-R e(r-z) / 2 \ell}+\frac{2}{R e} \frac{\ell}{r}\left(e^{-R e(r-z) / 2 \ell}-1\right)\right) .
$$

It is easily checked that as $R e \rightarrow 0$ this recovers the far-field displacement due to a Stokesian squirmer. Using this streamfunction we obtain numerically $\Delta_{\lambda}(a, b)$ for different values of $R e$ and summarise the results for the effective diffusivity in figure 7(b). For small $R e$, we recover the Stokes value of the effective diffusivity; for larger values of $R e$, the effective diffusivity drops off as $R e^{-0.61}$ due to smaller particle displacements. 


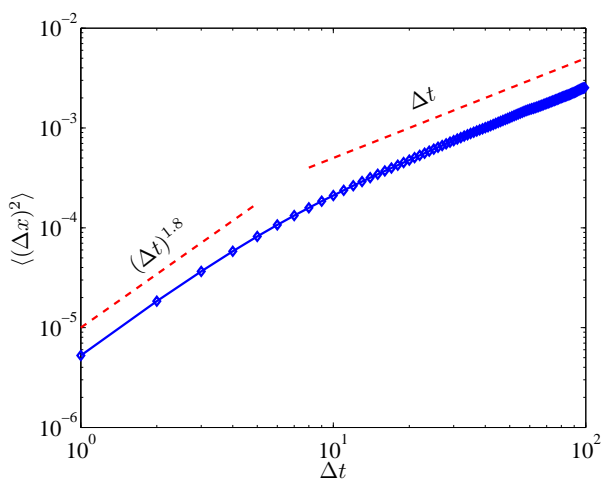

(a)

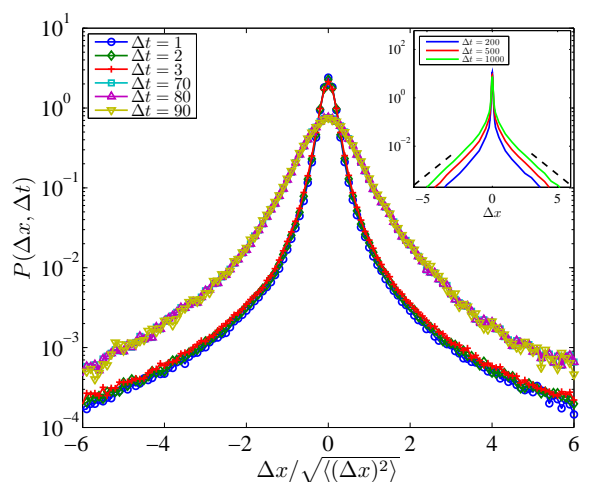

(b)

Figure 8. (a) Growth of mean-squared displacement $\left\langle(\Delta x)^{2}\right\rangle$ with time, with superdiffusive (short-time) and diffusive (long-time) regimes. (b) Rescaled $x$-displacement distributions in the two regimes, showing the collapse after scaling. Inset: unscaled PDFs with exponential tails (6.1).

\section{PDF of displacements}

We close with some comments regarding the typical size of displacements imparted by the squirmers. In a recent letter, Leptos et al. (2009) measured experimentally the probability distribution function (PDF) of displacements of a target particle due to repeated 'kicks' by swimming micro-organisms. They observe that, unlike in Einstein's theory of Brownian motion, the PDF is non-Gaussian with clear exponential tails. We now show that squirmers also exhibit such tails, due to the stagnation points at their surface.

The large displacements for squirmers are due to particles that lie very close to the axis of motion, i.e., with $a \ll \ell$. For small $a$, the displacement function can be wellapproximated with a 'top-hat' function as in (2.7). The probability of $\Delta_{\lambda}(a, b)$ being larger than a given value $\delta$ is then

$$
P\left(\Delta_{\lambda}>\delta\right)=\frac{1}{V} \int_{\Delta_{\lambda}(a, b)>\delta} \pi a \mathrm{~d} a \mathrm{~d} b=\frac{\lambda}{V} \int_{\Delta(a)>\delta} \pi a \mathrm{~d} a .
$$

For $a \ll \ell$, the squirmer displacement $\Delta(a)$ diverges as $C \ell \log (\ell / a)$, where $C$ is a numerical constant. Hence, the contribution for large $\delta$ comes from small $a$, and we have

$$
P\left(\Delta_{\lambda}>\delta\right) \approx \frac{\lambda}{V} \int_{C \ell \log a^{-1}>\delta} \pi a \mathrm{~d} a=\frac{\pi \lambda}{2 V} \mathrm{e}^{-2 \delta / \ell C},
$$

which exhibits exponential tails, as in Leptos et al. (2009). A simple argument using large-deviation theory shows that the PDF of a superposition of such exponential tails retains the same exponential tails.

To make contact with Leptos et al. (2009), we statistically analyse displacements for our biomixing model via particle simulations. We examine the displacement histograms for different sampling intervals $\Delta t$ up to 90 , over which each swimmer travels a distance $U \Delta t$. Figure 8(a) shows the mean-squared displacement $\left\langle(\Delta x)^{2}\right\rangle$ as a function of time: for short times, this grows as $(\Delta t)^{1.8}$, in good accordance with the superdiffusive regime observed by $\mathrm{Wu} \&$ Libchaber (2000): they found an exponent between 1.5 and 2. For longer times, we recover a linear diffusive regime, as expected, with slope given by our theoretical prediction $2 \kappa$. Figure $8(\mathrm{~b})$ shows that the incremental displacement probability distribution along the $x$-axis, $P(\Delta x, \Delta t)$, rescales to a single distribution function in terms of the similarity variable $\Delta x / \sqrt{\left\langle(\Delta x)^{2}\right\rangle}$ in each of the two scaling regimes. However, note that the exponential tails (6.1) are independent of $\Delta t$, and are only visible 
in simulations after integrating for a long time (see inset in figure 8(b)). Thus, it is not clear if these are related the the exponential tails observed by Leptos et al. (2009).

The authors are grateful to Keith Moffatt for suggesting the wake transport calculation, to Eric Kunze for pointing out that molecular diffusion would limit the wake transport, and to the Institute for Mathematics and its Applications (supported by NSF) for its hospitality. SC was supported by NSF under grant DMS-0507615, J-LT under grant DMS-0806821.

\section{REFERENCES}

BlAke, J. R. 1971 A spherical envelope approach to ciliary propulsion. J. Fluid Mech. 46, $199-208$.

Darwin, C. G. 1953 Note on hydrodynamics. Proc. Camb. Phil. Soc. 49 (2), 342-354.

Dewar, W. K., Bingham, R. J., Iverson, R. L., Nowacek, D. P., St. Laurent, L. C. \& Wiebe, P. H. 2006 Does the marine biosphere mix the ocean? J. Mar. Res. 64, 541-561.

Dombrowski, C., Cisneros, L., Chatkaew, S., Goldstein, R. E. \& Kessler, J. O. 2004 Self-concentration and large-scale coherence in bacterial dynamics. Phys. Rev. Lett. 93 (9), 098103.

Drescher, K., Leptos, K., Tuval, I., Ishikawa, T., Pedley, T. J. \& Goldstein, R. E. 2009 Dancing volvox: hydrodynamic bound states of swimming algae. Phys. Rev. Lett. 102, 168101.

Drescher, K. D., Goldstein, R. E., Michel, N., Polin, M. \& Tuval, I. 2010 Direct measurement of the flow field around swimming microorganisms. Phys. Rev. Lett. 105, 168101.

Dunkel, J., Putz, V. B., Zaid, I. M. \& Yeomans, J. M. 2010 Swimmer-tracer scattering at low Reynolds number. Soft Matter 6, 4268-4276.

Einstein, A. 1956 Investigations on the Theory of the Brownian Movement. New York: Dover.

Guasto, J. S., Johnson, K. A. \& Gollub, J. P. 2010 Oscillatory flows induced by microorganisms swimming in two-dimensions. Phys. Rev. Lett. 105, 168102.

Hernandez-Ortiz, J. P., Dtolz, C. G. \& Graham, M. D. 2006 Transport and collective dynamics in suspensions of confined swimming particles. Phys. Rev. Lett. 95, 204501.

Ishikawa, T. \& PeDley, T. J. 2007 Diffusion of swimming model micro-organisms in a semidilute suspension. J. Fluid Mech. 588, 437-462.

Ishikawa, T., Simmonds, M. P. \& Pedley, T. J. 2006 Hydrodynamic interaction of two swimming model micro-organisms. J. Fluid Mech. 568, 119-160.

Katija, K. \& Dabiri, J. O. 2009 A viscosity-enhanced mechanism for biogenic ocean mixing. Nature 460, 624-627.

Leptos, K. C., Guasto, J. S., Gollub, J. P., Pesci, A. I. \& Goldstein, R. E. 2009 Dynamics of enhanced tracer diffusion in suspensions of swimming eukaryotic microorganisms. Phys. Rev. Lett. 103, 198103.

Lighthill, M. J. 1952 On the squirming motion of nearly spherical deformable bodies through liquids at very small Reynolds numbers. Comm. Pure Appl. Math. 5, 109-118.

Maxwell, J. C. 1869 On the displacement in a case of fluid motion. Proc. London Math. Soc. s1-3 (1), 82-87.

Oseen, C. W. 1910 Über die Stokessche formel und über eine verwandte aufgabe in der hydrodynamik. Ark. Mat. Astr. Fys. 6 (29).

Saintillian, D. \& Shelley, M. J. 2007 Orientational order and instabilities in suspensions of self-locomoting rods. Phys. Rev. Lett. 99, 058102.

Thiffenult, J.-L. \& Childress, S. 2010 Stirring by swimming bodies. Phys. Lett. A 374, 3487-3490.

Underhill, P. T., Hernandez-Ortiz, J. P. \& Graham, M. D. 2008 Diffusion and spatial correlations in suspensions of swimming particles. Phys. Rev. Lett. 100, 248101.

Wu, X.-L. \& Libchaber, A. 2000 Particle diffusion in a quasi-two-dimensional bacterial bath. Phys. Rev. Lett. 84, 3017-3020. 